\title{
Cross-sectional study of rheumatoid arthritis treatment in a university hospital
}

\author{
W T FRIESEN, ${ }^{1}$ Y A HEKSTER, ${ }^{2}$ L B A VAN DE PUTTE, ${ }^{3}$ AND \\ F W J GRIBNA U
}

From the ${ }^{1}$ School of Pharmacy, University of Tasmania, Hobart, Australia; and Departments of ${ }^{2}$ Clinical Pharmacy, ${ }^{3}$ Rheumatology, and ${ }^{4}$ Pharmacology, Sint Radboud University Hospital, Nijmegen, the $\vec{\odot}$ Netherlands

SUMMARY Drug prescribing patterns for the management of inpatients and outpatients with $\dot{\omega}_{\dot{\omega}}^{\infty}$ rheumatoid arthritis (RA) were investigated. The population of patients resembled published $N$ epidemiological descriptions of RA patients with respect to age and sex distribution. Multiple을 drug therapy was common in the treatment of both hospitalised and clinic patients. $90 \%$ of all $\overrightarrow{ }$

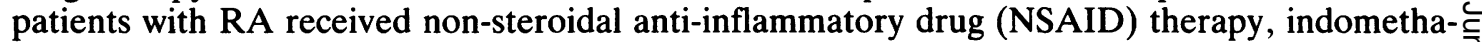

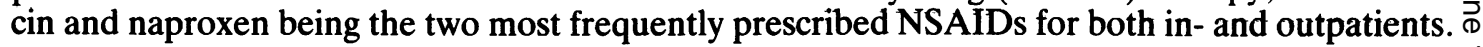
The vast majority of inpatients $(85 \%)$ and outpatients $(79 \%)$ received slow-acting antirheumatic $\overrightarrow{0}$ drug (SAARD) treatment. $13 \%$ of hospitalised patients received $\mathrm{H} 2$-antagonist drugs in addition to their NSAIDs. A high proportion of inpatients $(46 \%)$ received oral corticosteroids in the management of their rheumatoid arthritis, while only $15 \%$ of clinic patients were prescribed $\leqq$ corticosteroids.

Key words: antirheumatic drugs, drug utilisation, drug prescribing, non-steroidal anti- $\frac{\stackrel{\Phi}{\varrho}}{2}$ inflammatory drugs, slow-acting antiarthritic drugs, outpatients, inpatients.

The prevalence of chronic disease in modern industralised nafions is increasing, and among these intractable conditions rheumatoid arthritis stands out as a major cause of multiple medical problems. ${ }^{1}$ An estimated $1-3 \%$ of the population is affected by this disease, while about two-thirds of cases suffer significant social and economic disadvantages. ${ }^{12}$

Although some therapeutic measures appear to improve the clinical picture, the influence of drug therapy on the course of the disease is still uncertain. ${ }^{23}$ The twofold goals of treatment are well established, ${ }^{4}$ i.e., firstly, suppression of pain and inflammation with non-steroidal anti-inflammatory drugs (NSAID) and secondly, induction of remission of the disease with disease modifying drugs if the disease progresses. There is a bewildering array of NSAIDs available, and by comparison the repertoire of disease modifying slow-acting antirheumatic drugs (SAARD) is small.

Accepted for publication 4 December 1984.

Correspondence to $\mathrm{Dr} \mathrm{W} T$ Friesen, School of Pharmacy, University of Tasmania, PO Box 252C, Hobart, Tasmania 7001, Australia.
Considerable differences exist in prescribing practices, for example between countries ${ }^{5-7}$ and between $\stackrel{\overrightarrow{0}}{2}$ hospital and community-based practice. ${ }^{8}$ Although data are available on the drug utilisation of different classes of antiarthritic drugs, only limited information is available on prescribing practices specifically for rheumatoid arthritis.

This paper describes the results of a study $\rightarrow$ establishing baseline data on the drug treatment patterns for rheumatoid arthritis patients in the $N$ rheumatology ward and the rheumatology outpatient clinic of a Dutch university hospital.

\section{Patients and methods}

INPATIENT DATA COLLECTION

Patient data

All patients admitted to the rheumatology ward $\frac{7}{0}$ over a one-year period (June 1983 to May 1984) $\stackrel{\circ}{\mathbb{D}}$ were surveyed retrospectively. Patient details such $\frac{\mathbb{}}{\Phi}$ as age, sex, diagnosis details, and duration of $\stackrel{\mathbb{Q}}{\varrho}$ hospitalisation were determined from medical records. Only patients with probable, definite, or $\delta$ 
classical rheumatoid arthritis according to the American Rheumatism Association (ARA) criteria9 were admitted to the survey.

\section{Drug prescribing data}

Individual medication registration sheets are stored in the pharmacy department by ward specialty (i.e., rheumatology) and per time period (quarter). Drug prescribing details were compiled directly from these sheets as follows: antiarthritic drug or drug combinations, prescribed daily dosages, and all coprescribed drugs for each patient.

\section{OUTPATIENT DATA COLLECTION}

\section{Patient data}

A one-month prospective survey of rheumatoid arthritis patients treated in the Sint Radboud Hospital Outpatient Clinic was conducted. Only patients with definite or classical rheumatoid arthritis $^{9}$ were admitted to the survey. The data represent a cross-sectional definition of a clinic population. The following patient information was compiled: age, sex, and the current patient disability status based on the ARA functional class ${ }^{10}$ as assessed by the physician during the consultation.

\section{Drug prescribing data}

At the time of the consultation the drug prescribing data were recorded as follows: antiarthritic drug or drug combinations, dosage form, prescribed daily dosage, frequency, and all coprescribed drugs. Patterns of drug prescribing of individual drugs and particular drug combinations were then determined.

\section{Results}

\section{PATIENT CHARACTERISTICS}

\section{Inpatients}

A total of 166 patients were admitted to the rheumatology ward during the 12-month survey period. Of these, 108 patients $(65 \%)$ had a diagnosis of probable, definite, or classical rheumatoid arthritis and were included in the study. The age and sex distribution of the RA patients admitted to the rheumatology ward is shown in Fig. 1a. The duration of hospitalisation averaged 34.6 days and ranged between two and 206 days. The female: male ratio for inpatients is $2 \cdot 2: 1$.

\section{Outpatients}

A total of 153 definite or classical rheumatoid arthritis patients treated in the outpatient clinic during the month of June 1984 were surveyed. The age and sex distribution of these patients is shown in Fig. 1b. The female: male ratio for outpatients is 2.8:1.

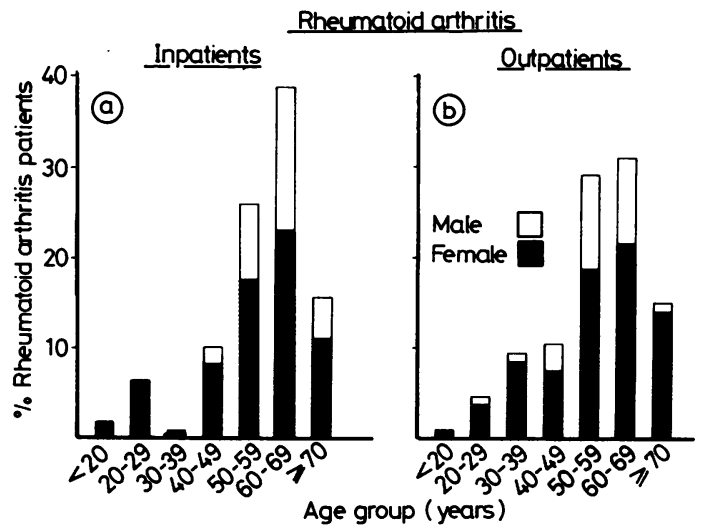

Fig. 1 (a) The age and sex distribution of rheumatoid arthritis inpatients admitted to the rheumatology ward during a 12-month period (June 1983-May 1984). $n=108$. (b) The age and sex distribution of rheumatoid arthritis outpatients treated in the rheumatology clinic during a one-month period. $n=153$.

The disability distribution of outpatients by age and sex according to the ARA functional capacity rating is shown in Fig. 2. It can be seen that the majority of patients fall into disability rating 2 , and no patient under the age of 40 had a disability rating of 3 . No patient had a disability rating of 4 .

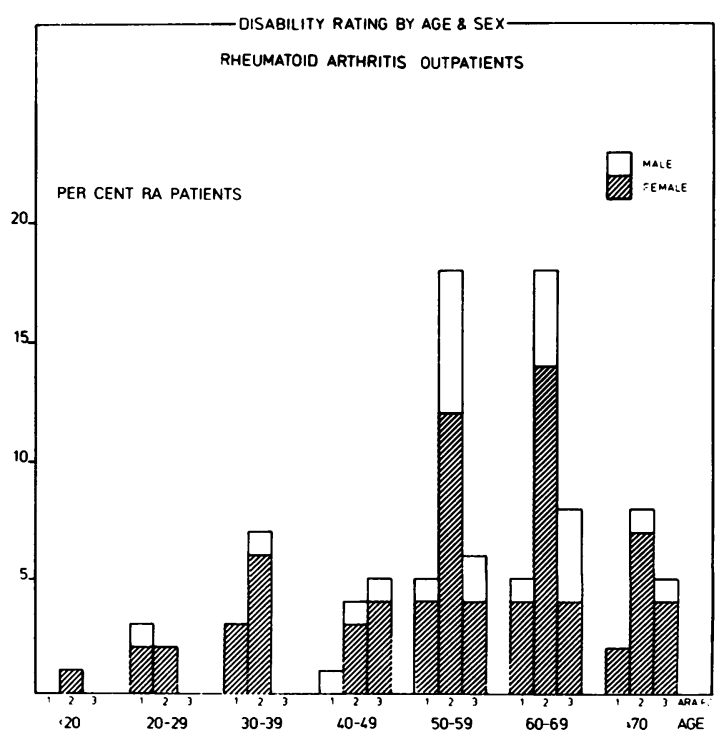

Fig. 2 The disability rating (ARA functional class) by age and sex for rheumatoid arthritis outpatients treated in the rheumatology clinic during a one-month period. $n=153$. 
DRUG UTILISATION DATA

Patterns of drug treatment

Fig. 3a outlines the current use of antirheumatic drug therapy for rheumatoid arthritis inpatients, indicating that polypharmacy is a common practice in the management of a hospitalised RA population. About $90 \%$ of the RA inpatients received NSAID therapy $(10 \%$ receiving a two-drug NSAID combination usually as one drug administered orally and the second one rectally at night; see Fig. 4a). $11 \%$ of the patients did not receive any NSAID therapy during their hospital stay.

Fig. 3a also shows that the vast majority of inpatients $(85 \%)$ received specific remittive therapy in the form of slow-acting antirheumatic drugs, and that almost half of the inpatients $(46 \%)$ received oral corticosteroid therapy (usually in a $5.0-7.5 \mathrm{mg}$ daily maintenance dose of prednisolone) during their hospital stay. About one-third of all RA inpatients $(36 \%)$ were treated with a NSAID+SAARD + oral corticosteroid combination, while an additional $40 \%$ of patients were prescribed NSAID+SAARD drug regimens. Only $1 \%$ of admitted patients had no specific antirheumatic therapy.

Fig. $3 b$ outlines the use of antirheumatic drug therapy for rheumatoid arthritis outpatients in the rheumatology clinic during the sample period. Like the inpatients $90 \%$ of these patients also received

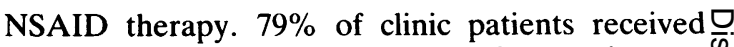
SAARD therapy, and the most frequently pre-: scribed drug regimen ( $63 \%$ of clinic patients) was $\overrightarrow{\overline{\vec{m}}}$ NSAID+SAARD combination therapy. $15 \%$ of allo clinic patients were prescribed oral corticosteroids in $\frac{\mathrm{C}}{\sigma}$ addition to NSAID or SAARD therapy, or both. A $\overline{\bar{c}}$ further $15 \%$ of outpatients received NSAID drugs $\widehat{\Phi}$ alone. $4 \%$ of patients received SAARD therapy alone, while only $3 \%$ of patients were not prescribed ${ }^{\infty}$ any specific antirheumatic treatment.

\section{NSAID prescribing data}

Fig. 4a presents a complete summary of NSAIDฏ prescribing frequency for inpatients. Indomethacin? was the most frequently prescribed NSAID (36\% of $\rightleftharpoons$ patients), and together with naproxen ( $24 \%$ of patients) these two drugs accounted for about two- $N$ thirds of the NSAIDs prescribed. $9 \%$ of hospitalised。 patients received piroxicam, while only $5 \%$ of ${ }^{\supset}$ patients were prescribed ibuprofen.

Fig. 4b shows the frequency distribution of NSAID prescribing for outpatients. The largest ${ }^{\Phi}$ single NSAID used was indomethacin $\left(41 \%\right.$ of ${ }_{\infty}$ patients), and together with naproxen $(22 \%$ of patients) these two drugs made up about $70 \%$ of the total use of NSAIDs in clinic patients. Ibuprofens was prescribed for $12 \%$ of outpatients, while newer agents accounted for a further $14 \%$ of NSAID usage.
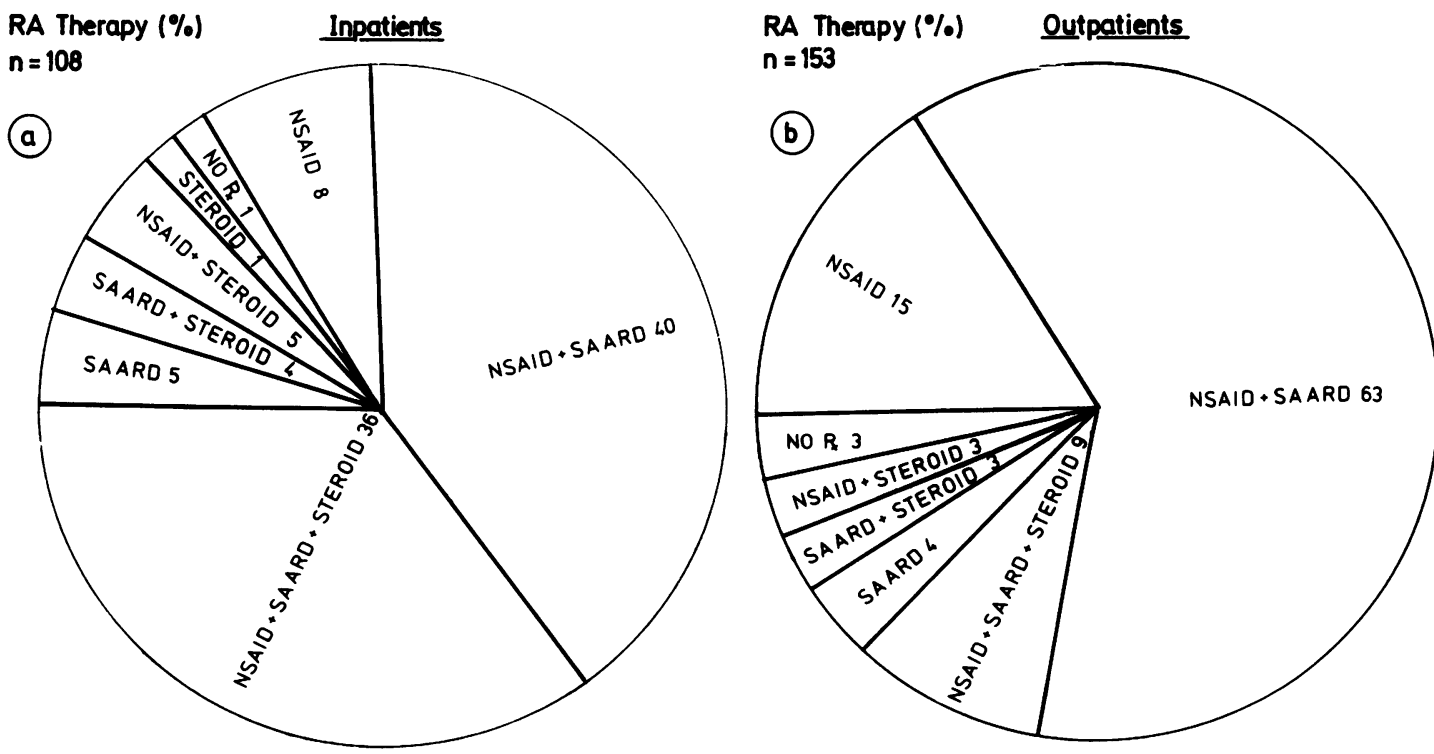

Fig. 3 Prescribing pattern of antirheumatic drug usage for the treatment of $R A(a)$ inpatients and (b) outpatients. The numbers refer to the percentage of patients receiving a particular drug category or combination. 

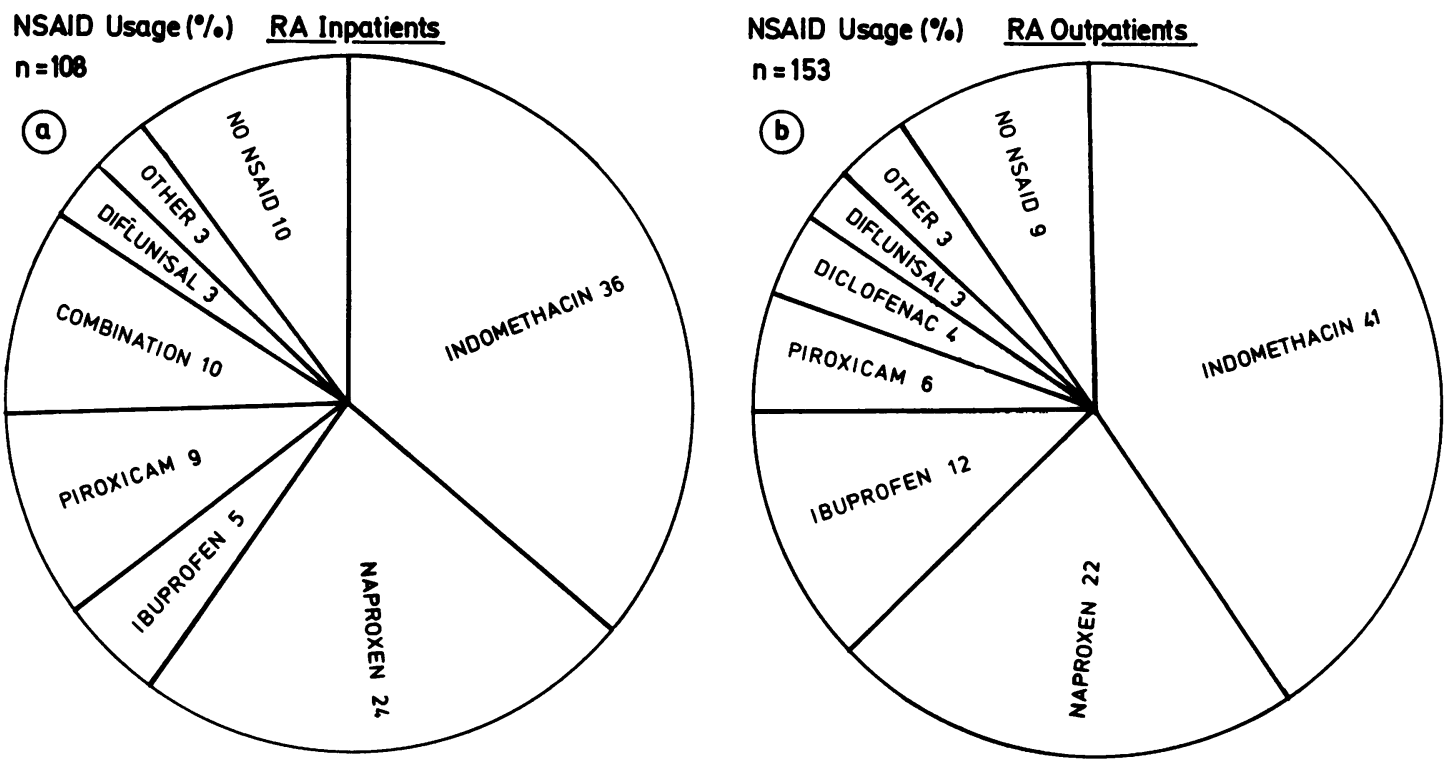

Fig. 4 Prescribing pattern of non-steroidal anti-inflammatory drug (NSAID) usage for the treatment of rheumatoid arthritis inpatients and outpatients. The numbers refer to the percentage of patients receiving a particular drug or combination.

SAARD prescribing data

The prescribing frequency of the SAARDs for inpatients, which from Fig. 3a can be seen to be used mostly in multiple drug therapy, is summarised in
Fig. 5a. Azathioprine had the highest usage $(38 \%$ of SAARD-treated patients), followed by aurothioglucose $(34 \%)$, and D-penicillamine $(26 \%)$.

The outpatient prescribing frequency of indi-

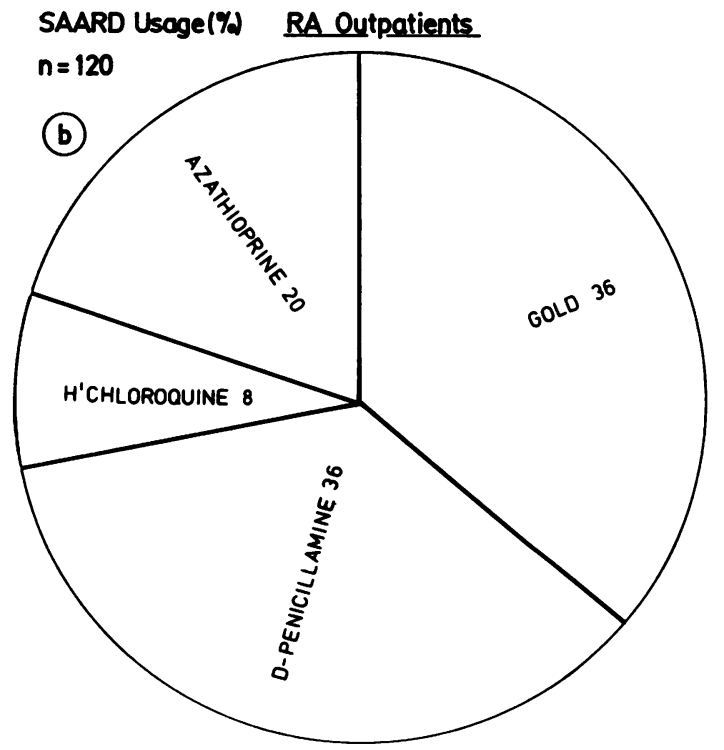

Fig. 5 Prescribing pattern for slow-acting antiarthritic drug (SAARD) usage for the treatment of rheumatoid arthritis inpatients and outpatients. The numbers refer to the percentage of patients receiving a particular drug.

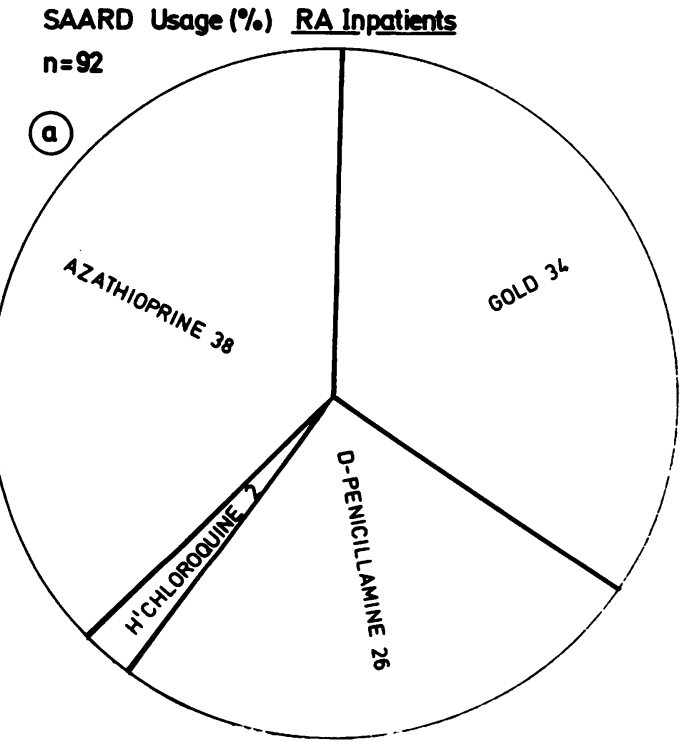


Table 1 Rheumatoid arthritis coprescribing data for inpatients

\begin{tabular}{ll}
\hline Drug combination & $\begin{array}{l}\text { Percentage of patients } \\
\text { (no. of patients) }\end{array}$ \\
\hline NSAID + cimetidine & $10 \cdot 2(11)$ \\
NSAID + cimetidine + antacid & $2 \cdot 8(3)$ \\
NSAID + antacid & $14 \cdot 8(16)$ \\
NSAID + other GI therapy & $1 \cdot 9(2)$ \\
NSAID + iron supplement & $17 \cdot 6(19)$ \\
NSAID + benzodiazepine & $38 \cdot 9(42)$ \\
NSAID + diuretic & $10 \cdot 2(11)$ \\
NSAID + analgesic & $45 \cdot 4(49)$ \\
\hline
\end{tabular}

vidual SAARDs used principally in combination therapy is shown in Fig. 5b. Aurothioglucose and Dpenicillamine were prescribed in equal frequency ( $36 \%$ of SAARD-treated patients each), while in contrast to the inpatient prescribing pattern, azathioprine accounted for only $20 \%$ of SAARD usage.

\section{Additional prescribing data}

Table 1 outlines the principal drugs coprescribed with antirheumatic therapy for inpatients. It can be seen that $13 \%$ of hospitalised patients received a combination of cimetidine and NSAID treatment, and overall about $30 \%$ of patients received some form of drug therapy to control gastric symptoms while on NSAID treatment.

A significant proportion of inpatients (17.6\%) received iron supplementation, and over one-third of admitted patients with RA $(38.9 \%)$ received benzodiazepine medication.

A large proportion of hospitalised patients $(45.4 \%)$ received analgesic therapy in addition to their antirheumatic drugs. About $10 \%$ of patients were taking a diuretic in conjunction with NSAID drugs.

There was very little in the way of coprescribed drug therapy in the clinic patients. Only $3 \%$ of patients received $\mathrm{H} 2$-antagonist drugs and only $7 \%$ of outpatients were prescribed benzodiazepines in addition to their antirheumatic treatment.

Prescription instructions for clinic patients were also noted. About $19 \%$ of patients received nonspecific instructions regarding the dosage of their treatment, i.e., $8 \%$ of prescriptions were to be 'used as required', and $11 \%$ of prescriptions gave patients instructions to use the NSAID treatment within a dosage range.

\section{Discussion}

Our sample statistics were consistent with epidemiological data on age and sex distribution imp? rheumatoid arthritis, ${ }^{11} 12$ there being a two to thre times greater prevalence in females than in males fof both inpatients and clinic patients. The majority of cases were over the age of 50 (about $80 \%$ o inpatients and about $75 \%$ of outpatients). Among younger patients with rheumatoid arthritis the overe whelming majority were female, as no male patient under the age of 40 were admitted to the rheumatology ward during the one-year survey period, and only $10 \%$ of patients under the age of 40 among the RA outpatients were male.

The functional capacity data for the clinic patient彦 indicate that a relatively lower proportion of out patients $(24 \%)$ developed severe handicap (categories 3 or 4 in the ARA classification) than was reported for clinic-treated patients elsewhere. Rasker and $\operatorname{Cosh}^{13}$ reported $45 \%$ of their patients to be in categories 3 or 4 ; however, no reference was madeto relationships between functional class and treat ment. The widespread use of second-line drugs ind our treatment regimens and the ready access to orthopaedic surgery could well account for a disabilio ity profile in this study, which is similar to that reported for rheumatoid arthritis patients treated in community practice ${ }^{8}$ who would not be expected to have such a severe disease as clinic-treated patients.

Published data on prescribing patterns foo rheumatoid arthritis are extremely limited. Informa tion concerning NSAID drug utilisation is available $\overrightarrow{\overrightarrow{0}}$ but little of it is linked to morbidity (i.e., to specific forms of arthritis). Ibuprofen is currently the most widely prescribed NSAID in Britain, ${ }^{7}$ the Netherlands, ${ }^{14}$ and in the USA, ${ }^{5}$ excluding aspirin.$\frac{0}{2}$ Naproxen is the most widely prescribed NSAID in the Scandinavian countries ${ }^{6}$ and in Australia. ${ }^{15}$

In a more specific audit in a rheumatology clinic, ${ }^{16}$ where $77 \%$ of the patients had RA, indomethacin naproxen, and ibuprofen accounted for $47 \%$ of theo NSAID prescriptions. In an Australian community based study dealing specifically with rheumatoido. arthritis, ${ }^{\overline{8}}$ naproxen $(25 \%)$, aspirin $(25 \%)$, and in domethacin $(19 \%)$ together accounted for $69 \%$ of the NSAID usage. Our study showed indomethacin 0 and naproxen to be the first and second ranking drugs prescribed for both inpatients $(67 \%$ total) ando outpatients $(69 \%$ total), with ibuprofen having o relatively minor role and aspirin virtually not prescribed.

Statistics on consumption of SAARDs and theiro combination with other agents are even more scarce. Data on drug utilisation on national levels $\mathbb{\mathbb { Q }}$ for SAARDs and corticosteroids ${ }^{6}$ are of limited $\mathbb{Q}_{\mathbb{Q}}$ value because they are not linked to diagnosis. In $\mathrm{a}_{0}$ British study, ${ }^{16}$ however, $32 \%$ of outpatients received gold therapy and $17 \%$ received 
Table 2 Prescribed daily doses (PDD) versus defined daily doses (DDD) for non-steroidal anti-inflammatory drugs in the treatment of rheumatoid arthritis

\begin{tabular}{|c|c|c|c|c|c|c|c|}
\hline \multirow[t]{3}{*}{ Drug } & \multicolumn{3}{|c|}{ Inpatients } & \multicolumn{4}{|l|}{ Outpatients } \\
\hline & \multirow[t]{2}{*}{$n$} & \multicolumn{2}{|c|}{$P D D$ (mg) } & \multirow[t]{2}{*}{$D D D(m g)$} & \multirow[t]{2}{*}{$n$} & \multicolumn{2}{|c|}{$P D D$ (mg) } \\
\hline & & Mean & Range & & & Mean & Range \\
\hline Indomethacin & 45 & 125 & $50-300$ & 100 & 44 & 45 & $25-200$ \\
\hline Naproxen & 33 & 780 & $500-1000$ & 500 & 24 & 653 & $125-1000$ \\
\hline Piroxicam & 10 & 18 & $10-30$ & 20 & 6 & 23 & $20-30$ \\
\hline Ibuprofen & 7 & 771 & $400-800$ & 800 & 13 & 1233 & $400-1600$ \\
\hline
\end{tabular}

penicillamine. Our study showed a similar prescribing rate for gold ( $28 \%$ of patients) but a higher use of Dpenicillamine $(28 \%)$ in the outpatient clinic.

No comparative data have been found for multiple-drug prescribing except for the communitybased study ${ }^{8}$ which showed a considerably lower incidence of polypharmacy than in our hospitalbased study. We found that on average about $82 \%$ of patients received SAARD therapy $(85 \%$ of inpatients, $79 \%$ of outpatients), compared with only $18 \%$ in the community-based study. Although $46 \%$ of inpatients received oral corticosteroids in the management of acute phases of rheumatoid arthritis, only $15 \%$ of outpatients were prescribed oral corticosteroids. This compares with $23 \%$ of patients receiving corticosteroids therapy in the communitybased study. ${ }^{8}$ Recently Million et al. ${ }^{17}$ have suggested that judicious use of steroids can greatly improve the mobility and welfare of many rheumatoid arthritis patients without the severe complications usually associated with steroid therapy. The need to prescribe H2-antagonist therapy in conjunction with NSAIDs was considerably higher in inpatients $(13 \%)$ than in outpatients $(3 \%)$. This may be reflected in the generally higher prescribed daily doses of NSAIDs (Table 2) employed in the treatment of inpatients than in clinic patients.

A comparison of prescribed daily doses (PDD) between inpatients and outpatients (Table 2) shows that inpatients received higher doses of the principal NSAIDs, indomethacin and naproxen, well above the defined daily dose (DDD), an international unit of comparison for drug utilisation studies, being the average maintenance dose for the principal indication of a drug per day. ${ }^{18}$ This pattern of increased doses in inpatients may be expected because these patients would probably be hospitalised due to an acute exacerbation of rheumatoid arthritis, and higher doses of NSAID could be administered because, as inpatients, they could be more easily monitored for adverse effects than could clinic patients. Outpatients received doses of indometha- cin and naproxen which were closer to the DDDs, while ibuprofen and the non-formulary drug, piroxicam, were prescribed in less conservative doses, perhaps reflecting a confidence in their lower incidence of adverse effects.

This paper provides baseline data concerning the drug prescribing patterns in the management of both hospitalised and clinic-treated rheumatoid arthritis patients. With the benefit of an insight into current prescribing patterns gained from an audit such as this it should be possible to evaluate prescribing practices and to develop policies or algorithms for the management of rheumatoid arthritis.

The authors gratefully acknowledge the assistance of the medical staff in the rheumatology outpatients clinic, Dr F Speerstra, Dr L Santen, and Dr H Houben.

\section{References}

1 Meenan R F, Yelin E H, Nevitt M, Epstein W V. The impact of chronic disease. A sociomedical profile of rheumatoid arthritis. Arthritis Rheum 1981; 24: 544-9.

2 Scott D L, Couton B L, Chapman J H, Bacon P A, Popert A J. The long-term effects of treating rheumatoid arthritis. $J R$ Coll Physicians Lond 1983; 17: 79-85.

3 Wright W V, Amos R. Do drugs change the course of rheumatoid arthritis? $\mathrm{Br}$ Med $J$ 1981; 280: 964-6.

4 O'Duffy J D. Luthra H S. Current status of disease-modifying drugs in progressive rheumatoid arthritis. Drugs 1984; 27: 373-7.

5 Baum C, Kennedy D L, Forbes M B, Jones J K. Drug utilization in the US. 1982: Fourth Annual Review. Department of Health and Human Services, Division of Drug Experience, National Center for Drugs and Biologics, Food and Drug Administration, Bethesda, 1983.

6 Kass E. Drugs used in rheumatic diseases. In: Sakshaug S, Marit A, Hjort P F, Lunde P K M, Oydvin K, eds. Drug utilization in Norway during the 1970s: increases, inequalities, innovations. Oslo: Norwegian Medicinal Depot, 1983.

7 Freeborn S F, Gibson P, Clinch D. Prescribing patterns for NSAIDs in a typical urban area. Br J Pharm Pract 1983; 5: 20-5.

8 Owen S G. Friesen W T. Roberts M S. Flux W. Patient outcome and treatment data in a rheumatoid arthritic population (abstract). Australian Pharmaceutical Science Association Meeting, Melbourne, March 1984.

9 Ropes M W. Diagnostic criteria for rheumatoid arthritis: 1958 
revision by a committee of the American Rheumatism Association. Ann Rheum Dis 1959; 18: 49-53.

10 Stenbrocker O, Traeger C H, Batterman R C. Therapeutic criteria in rheumatoid arthritis. JAMA 1949; 140: 659-62.

11 Hochberg M C. Adult and juvenile rheumatoid arthritis: current epidemiological concepts. Epidemiol Rev 1981; 3: $27-41$.

12 Linos A, Worthington J W, O'Fallon W M, Kurland L T. The epidemiology of rheumatoid arthritis in Rochester, Minnesota. A study of incidence, prevalence and mortality. Am J Epidemiol 1980; 111: 87-98.

13 Rasker J J, Cosh J A. Cause and age at death in a prospective study of 100 patients with rheumatoid arthritis. Ann Rheum Dis 1981; 40: 115-20.
14 Zuidgeest L J B. Personal communication. Regional survey of $\frac{5}{\text { ? }}$ NSAID use in the Nijmegen and Tilburg areas from health insurance data.

15 Hall R C. Patterns of drug utilization - national and international aspects: drug use in Australia. Acta Med Scand 1982; 683 (suppl): 79-80.

16 Takavarasha L, Scott D L, Constable T J. A pharmacist's audit $\frac{\bar{\sigma}}{T}$ of antirheumatic drugs. Rheumatol Rehabil 1982; 21: 201-5.

17 Million R, Kellgren J H, Poole P, Jayson M I V. Long-term study of management of rheumatoid arthritis. Lancet 1984; i: 812-6.

18 Nordic Council of Medicines. Nordic Statistics on Medicines, Parts I and II. Statistical Reports on the Nordic Countries (1978-1980). Nos. 8 and 9, Uppsala. 1982. 